might well be a reflection of failures of the scientists themselves. It is not that the public does not understand science, he argues, rather that it has learnt not always to trust the opinions of scientists: "science is in a negative, if slowly unfolding, spiral of the self-destruction of its own public credibility." Now, how is corporate planning going to deal with that?

Kenneth Green is at the Manchester School of Management, University of Manchester Institute of Science and Technology, PO Box 88, Manchester M60 1QD, UK.

- Also for those concerned about the prospects of science in Britain comes British Science and Politics Since 1945 by Tom Wilkie. Published by Blackwell, this addition to the series 'Making Contemporary Britain' charts the history of British science and scientists in relation to government policy. Price is $£ 29.95$ (hbk), $£ 8.95$ (pbk).

\section{Living together}

\section{Brian Charlesworth}

Symblosis as a Source of Evolutionary Innovation: Speciation and Morphogenesis. Edited by Lynn Margulis and René Fester. MIT Press: 1991. Pp. 408. $\$ 37.50$, £33.75.

IT IS now universally accepted by biologists that the mitochondria and chloroplasts of eukaryotic cells are the descendants of bacteria-like organisms that took up residence within host cells, establishing an 'endosymbiotic' relationship. There is suggestive evidence from recent work on Chlamydomonas that the same is true of the centriolar apparatus. Lynn Margulis has played a large part in persuading biologists that such important features of eukaryote cells are the evolutionary product of endosymbiosis, and her contributions to this subject have provided a valuable addition to our understanding of the evolution of life. The purpose of this symposium volume is to bring together contributions from a diverse array of biologists to discuss whether symbiosis plays a major role in the generation of evolutionary novelty.

Symbiosis is defined here by Margulis as "association through a significant portion of the life history" between members of two different species. As many of the papers document, there is a wide range of examples of symbiosis, involving very different levels of intimacy between the partners. At one extreme, there are the mitochondria and chloroplasts, the genomes of which are much reduced in complexity compared with their ancestors and which are dependent on the host cell for many of the functions necessary for their survival. At an intermediate level, there are essentially obligate associations between partners, either intracellular or extracellular, such as the endomychorrizal fungi associated with the roots of many species of flowering plants. In other cases, one of the partners can be found either as freeliving or in association with the host, and the host has to be reinfected each generation. An example of this is the photobacterium associated with the light organs of gadiform fish. It is often difficult to distinguish between a parasitic and a mutualistic relationship, and critical data on fitness costs and benefits are often lacking. The start of a mutualism may often involve an exploitative relationship; conversely, mutualistic relationships may evolve into parasitic ones.

Two very different perspectives on the significance of symbiosis for general evolutionary biology are represented in this book. One, expounded lucidly by Richard Law and John Maynard Smith, is that symbiosis represents an interesting and important element in evolution, but in no sense offers a challenge to neodarwinian evolutionary theory. The other, vehemently stated by Margulis in the first chapter and espoused to varying extents by Robert Haynes, David Bermudes and Richard Back, Paul Nardon and Anne-Marie Grenier, Werner Schwemmler and Kris Pirozynski, is that neodarwinism is seriously incomplete, and that "the current practices of population biology and genetics must be obliterated by their own false assumptions" (Margulis, page 11). According to Margulis: "The standard textbooks on evolution catechize [sic] all species and higher taxa (genera, families, phyla) as having evolved in the same way: by the gradual accumulation of favourable mutations. Yet not a single example of the origin of such lower taxa (species) exists in the literature. Rather, the highest taxa (kingdoms and phyla) have evolved by acquisition of symbionts that have become hereditary."

This claim, if true, would certainly require a considerable reappraisal of evolutionary theory. There are, however, substantial grounds for doubting that it contains more than a grain of truth. In the first place, it is clear that symbiosis can allow a species to acquire a set of functionally integrated, adaptively useful characters in one step; for example, species of fish and nematodes have become luminescent by acquiring bacteria with elaborate pathways for light production. But unless a system of Chinese boxes is allowed, the ultimate source of the characters acquired by the host must be through the conventional darwinian process of the stepwise accumulation of individually advantageous changes.
Second, it is abundantly clear from many examples of symbiosis that the initial association sets the stage for a series of evolutionary adjustments by both partners. Again, there is no reason to suppose that this is anything other than a purely darwinian process. (One disappointing aspect of the book is the lack of discussion of the causes of some of these secondary events; for example, why should the nuclear genome have taken over so many of the functions of mitochondria and chloroplasts?) The properties conferred on the host by the original symbiosis were probably very different from those observed today.

Third, it is far from clear that symbiotic events in themselves are causally involved in either the splitting of lineages, or the evolution of the suite of characters that defines a major group. At the lowest level, the acquisition of a component of the gut flora by a species of insect or vertebrate does not in itself constitute the origin of a new species, unless it is accompanied by reproductive isolation from the rest of the population. Although there are some examples of hybrid sterility induced by endosymbionts, the evidence strongly supports the importance of nuclear genes in most cases of reproductive isolation that are susceptible to genetic analysis. At higher levels, there is no evidence that evolutionary events that are usually considered to be major are due to symbiosis: it seems unlikely, for instance, that the notochord was acquired in this way. Even though modern eukaryote cells contain endosymbionts of critical importance to their functioning, it is a mistake to identify the origin of the taxa concerned simply with the acquisition of the symbionts in question. Eukaryote cells, after all, have numerous important characteristics in addition to mitochondria. Darwinian evolution is the only tenable explanation of how such combinations of multiple characters can be built up.

The strength of this book is in the wealth of information on the natural history of symbiosis. Evolutionary biologists will certainly profit from this, although there are certain blind spots, such as the omission of any discussion of entities such as retroviruses and transposable elements, which surely meet Margulis's criterion for symbiosis. Its weakness lies in the exaggerated claims for symbiosis as the major source of evolutionary novelty. This may provoke unnecessary reluctance among mainstream evolutionists to think about the questions raised by symbiosis, which would surely by a pity.

Brian Charlesworth is in the Department of Ecology and Evolution, University of Chicago, 1101 East 57th Street, Chicago, IIlinois 60637-1573, USA. 